\title{
El Suple 2
}

\section{doi: 10.33264/rpa.201802-10 \\ Sandra Moreno Oyarzún, Alfredo Da Venezia Vásquez Escuela de Diseño Facultad de Arquitectura, Diseño y Artes Visuales}

\section{Resumen}

Proyecto que resume la búsqueda y las hipótesis de diseño visual obtenidas mediante la metodología de observación SUPLE desarrollada en la Escuela de Diseño de la Universidad UNIACC en Chile, se trata de la segunda experiencia documentada en la cual se obtienen soluciones a partir de la observación y recopilación de información en el propio entorno de los alumnos y profesores, en dispositivos domésticos, cotidianos y locales, en lugares donde a menudo no son evidentes, pero que se hacen visibles mediante el uso de una pauta de observación previa y consciente.

Palabras clave: identidad, diseño, doméstico, observación, descripción, territorio, educación, metodología, prótesis.

\begin{abstract}
Project that summarizes the search and visual design hypotheses obtained through the SUPLE observation methodology developed at the School of Design of the UNIACC University in Chile, it is the second documented experience in which solutions are obtained from observation and collection of information in the environment of students and teachers, in domestic, everyday and local devices, in places where they are often not evident but which are made visible by using a pattern of prior and conscious observation
\end{abstract}

Keywords: identity, design, domestic, observation, description, territory, education, methodology, prosthesis. 


\section{Introducción}

En el primer libro SUPLE se expone una hipótesis arriesgada; la educación puede abordarse desde una perspectiva diferente, no teórica ni tampoco creativista, sino que desde la cotidianeidad y realidad diaria de los mismos alumnos. En él se describe el proyecto y la forma en que los autores la desarrollan junto a un grupo de alumnos de la Escuela de Diseño de Universidad UNIACC.

Este nuevo libro es consecuencia de los resultados obtenidos y de la certeza de que además de arriesgado es un tema esencialmente amplio, que involucra una serie de análisis y posibilidades que van más allá de la educación del Diseño.

No era posible evadir este desafío, por eso la necesidad de profundizar el tema de investigación sobre el Suple, como se llama en Chile a cualquier dispositivo doméstico que nace de la urgencia de una reparación transitoria y que luego se eterniza.

Este SUPLE que no sólo representa un símbolo de lo conocido, de lo diario, sino que como elemento de diseño, le permite a un alumno construir sus observaciones y posteriormente conectar el aprendizaje desde su propio territorio, no sólo desde el territorio del profesor o desde la teoría. Proponiendo preguntas acerca de los objetos y saberes que le son propios al estudiante, para saber lo que el alumno conoce, nos permite, por una parte, valorar el conocimiento con que ingresa el estudiante, que éste se sienta valorado por su profesor y finalmente identificarse con los conocimientos que tiene y con los que incorporará posteriormente.

Este enfoque se ha ajustado al perfil de ingreso de los estudiantes que recibe la Escuela de Diseño UNIACC, donde en promedio poseen conocimientos limitados de arte, gráfica y diseño, que a su vez, son diferentes a los conocidos por los profesores, por lo tanto es esencial, comenzar desde el propio territorio del estudiante. Desde esta perspectiva, usar y darle la importancia necesaria se enfatiza y coincide con el modelo educativo UNIACC.

\section{Observar o no observar, esa es la cuestión}

La observación es el núcleo de este proyecto y del modelo Suple, es la metodología que permite a los alumnos comprender los desafíos de Diseño, compartirlos y problematizar lo que ven.

Desde el punto de vista del positivismo, observar es una forma de demostrar o negar una hipótesis que nos permitirá establecer o desechar una ley. Si interpretamos esta observación podremos conocer y entender los significados y el sentido que tiene cada una de nuestras propias acciones, las cuales constituyen precisamente los 
procesos que este proyecto espera de los participantes. La observación entonces sirve como nexo entre la teoría y la práctica, ya que a partir de la interpretación de lo observado es posible desarrollar propuestas para la transformación de esta realidad observada.

En este nuevo libro las observaciones de Diseño y las soluciones solicitadas son planteadas a alumnos con experiencia en el modelo Suple, por lo que una serie de preparaciones son acotadas a una problemática nueva y por supuesto a una solución nueva.

\section{La observación se plantea además como:}

- Activa, porque se observa pero también se actúa para transformar lo que se observa.

- Compartida, porque los resultados de ella, son compartidos en grupo, es decir entregan y reciben comentarios de los compañeros y profesores.

- Es un método, porque no es una simple técnica, implica una reflexión epistemológica y teórica.

\section{Objetivo General}

En base a la experiencia obtenida con la publicación SUPLE de noviembre 2017, se pretende como primer eje potencializar este nicho temático, como eje vinculante de los aprendizajes significativos, diferenciador y estratégico de la disciplina del diseño, tanto como la metodología educativa innovadora. En esta ocasión se incluirá un nuevo eje en las fichas de observación, que dicen relación con la reflexión y vinculación con el medioambiente a nivel nacional. Un segundo punto de apoyo es extender las relaciones disciplinarias con esta problemática con el fin de generar un puente en donde el diseño se propone como una disciplina pensante y no solo subsidiaria formal de otras con las cual tenemos fuertes vínculos como la literatura, el arte, la arquitectura y lo audiovisual.

\section{Objetivo General:}

- Sistematizar un modelo educativo centrado en el estudiante, de acuerdo a los lineamientos de UNIACC, en lo que refiere a un modelo centrado en el estudiante, basado en la observación y reflexión de lo conocido y cotidiano, para mejorar la enseñanza del Diseño.

\section{Específicos:}

- Validar un sistema de fichas de observación, que contengan un ítem de reflexión y analogías, que estimulen al estudiante a observar, analizar y 
concluir acerca de un elemento cotidiano y doméstico para extenderlo a una solución de problemas de Diseño.

- Recopilar información, acerca de los elementos cotidianos propios del entorno de los estudiantes de la Escuela de Diseño.

- Obtener material como registro fotográfico de elementos de Suple, para generar una publicación editorial, que una evidencia, de una metodología de Diseño, basada en la observación de lo cotidiano, que redunde en propuestas de soluciones de Diseño, con identidad nacional. Así mismo que este material editorial libro impreso, sea un elemento físico, de difusión e identidad de esta Escuela.

- Evidenciar a través de una muestra pequeña de un número de estudiantes y profesores de la escuela de diseño UNIACC de Santiago de Chile, cómo es posible levantar una información relevante en el diseño chileno con la mayor economía de medios y efectividad.

- Reflexionar y vincular las observaciones, con el medioambiente a nivel nacional y reforzar las relaciones con esta problemática a nivel latinoamericano.

Estas publicaciones tienen como objetivo principal, cimentar esta metodología en la educación del Diseño, basado en la observación y reflexión de los elementos que son propios a los estudiantes, usando herramientas de la antropología y de los diferentes sistemas educativos y métodos de observación cualitativa. La metodología consistirá en buscar en su habitar, los dispositivos domésticos que nacen de la urgencia de una reparación transitoria que se eterniza, partiendo desde la observación de lo cotidiano, de la identidad propia y nacional, de pequeños insights que no somos capaces de ver, a no ser, por la influencia de una pauta de observación consciente definida en una ficha de investigación.

La base de la publicación, es el trabajo con los alumnos de primer año y luego se extenderá a alumnos de segundo año, desde su entorno directo: el domicilio, lo que nos permitirá que el estudiante desde primer año reconozca su propio territorio como un escenario valorable (relación de sí mismo con el entorno y afianzamiento de su autoestima) que se plasmarán, bajo cierta metodología de estructuras conceptuales para observar, entregada por el docente a cargo de la investigación. En una futura edición y gracias a la primera publicación de Suple, la temática se complementará con textos y/o obras de los docentes profesionales de la escuela, como también algunos invitados externos provenientes de otros disciplinas a tratar el tema SUPLE a nivel nacional.

La comisión editorial evaluará y realizará una taxonomía para generar un material 
editorial que se traduzca en una publicación matriz, la que replicará bajo este concepto u otro, año tras año, como testimonio de la metodología, así como también, como identidad de la Escuela de Diseño UNIACC.

\section{Metodología}

\section{El Proceso de la experiencia}

A continuación presentamos la experiencia del proyecto Suple 2, que en esta edición, está vivenciada por los estudiantes del Taller Cuerpo de segundo año, que anteriormente realizaron la experiencia del proyecto Suple 1, cuando cursaban primer año y por 4 profesores de esta misma Escuela, invitados a descubrir, a través de la observación, problemáticas desde sus propias disciplinas.

Gráfico n¹: Organización del proyecto de observación

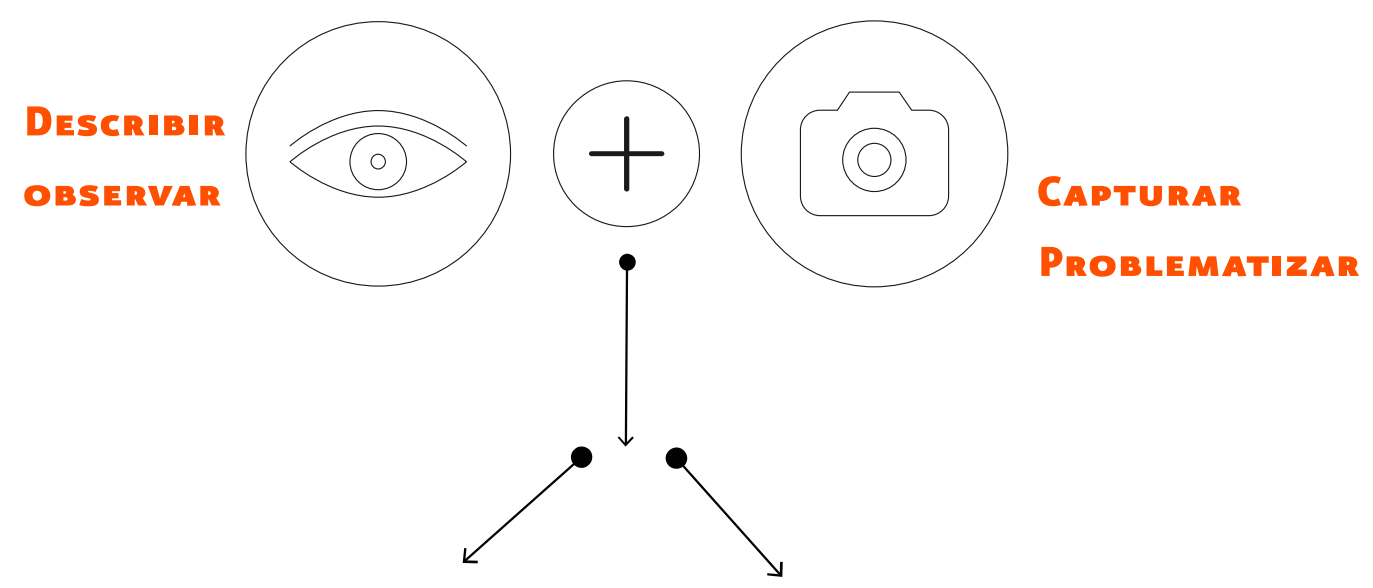

EXPERIENCIA ALUMNOS

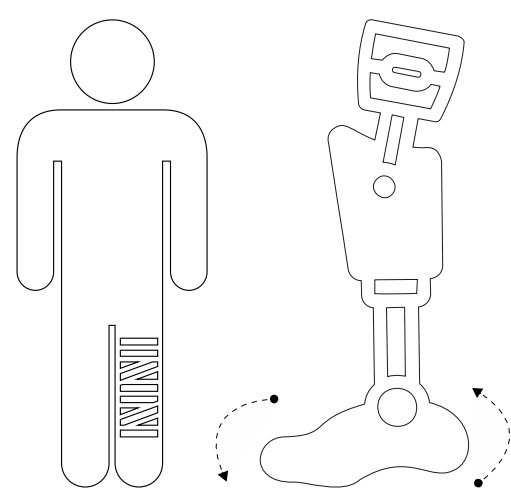

EXPERIENCIA PROFESORES
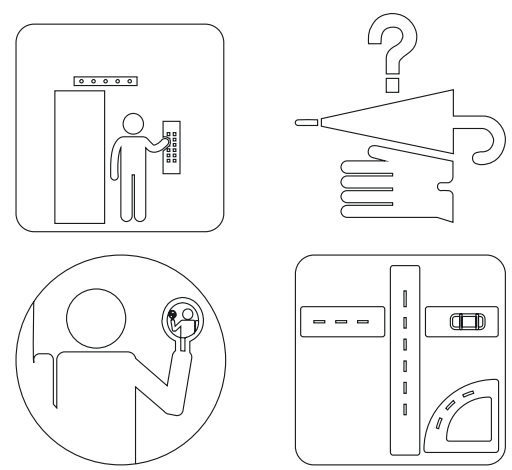

Nota: Infografía de los autores, basados en pictogramas de Noun Proyect (2018). 
Gráfico ${ }^{\circ}{ }^{2}$ : Proceso de aprendizaje con la metodología propuesta

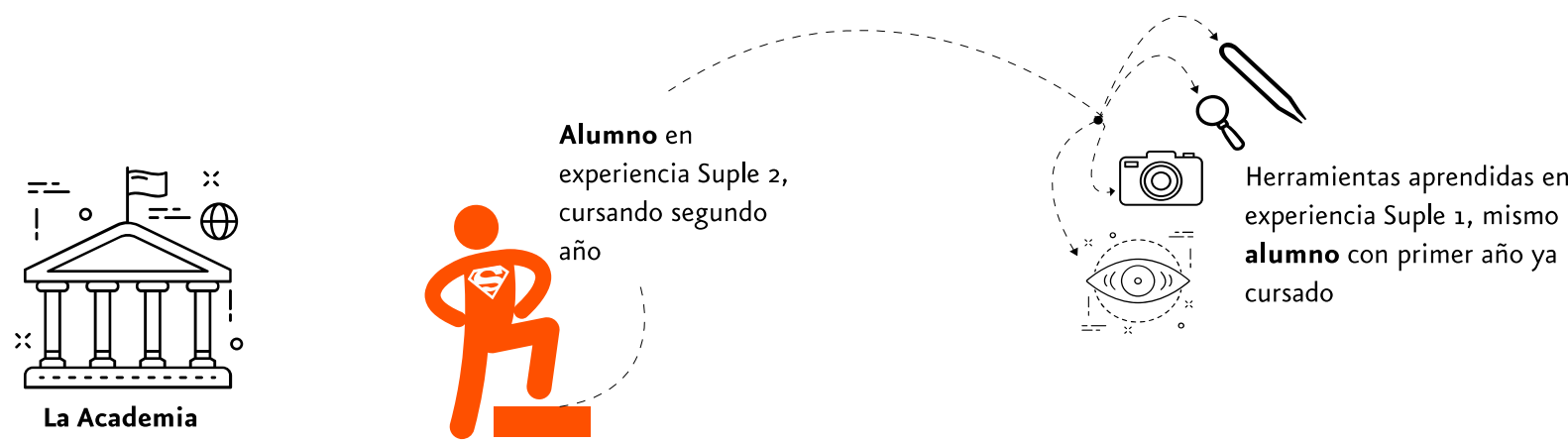

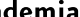

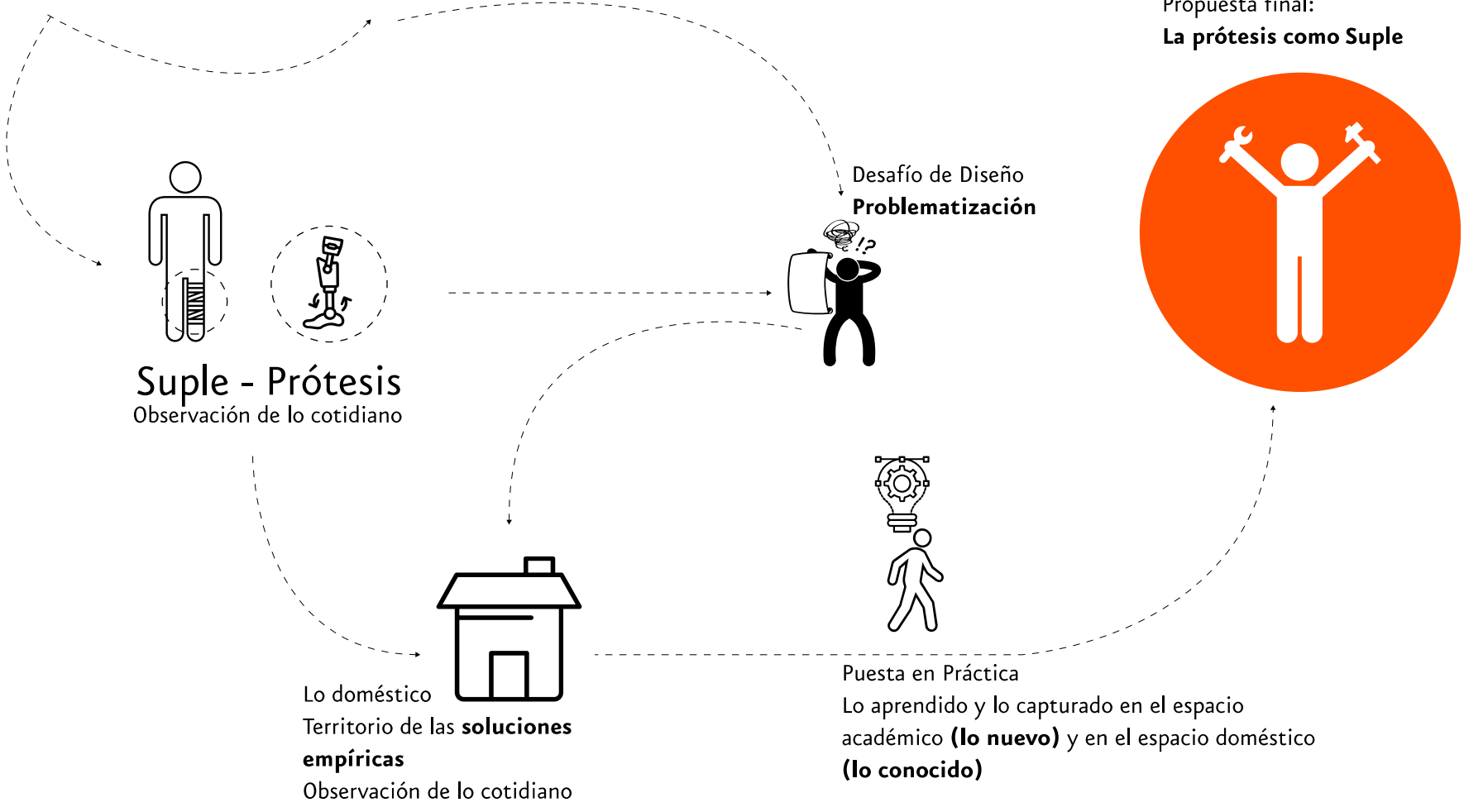

Propuesta final:

Nota: Infografía de los autores, basados en pictogramas de Noun Proyect (2018).

\section{El suple como prótesis, \\ la prótesis como suple}

En el año 2017 la Escuela de Diseño UNIACC, publicó el primer número de Suple, bajo el Concurso de Investigación Académica de la Universidad. La estrategia anexa a la investigación en sí, es generar por medio de los trabajos de alumnos y docentes de la Escuela, un manifiesto teórico/ práctico en base a textos y obras bajo este concepto 
tan relacionado con nuestra identidad que entendemos como Suple, resumido aquí como economía y territorio del diseño.

En el número publicado, Suple se define como: un objeto de reparación cotidiano, sin forma concreta, ni duración permanente, que se eterniza como solución para enfrentar condiciones mínimas y mayores en la reparación de problemas domésticos.

Bajo esta premisa se construyó un documento en base al investigación doméstica de estos dispositivos transitorios y vernáculos de los alumnos de primer año y su relación con lo académico en la esfera del diseño; hoy estos mismos alumnos desde el Taller de Cuerpo, en segundo año, investigan esta propiedad desde la relación que se puede generar del Suple como prótesis.

Según la RAE, prótesis se define en su primera acepción como:

1. f. Med. Pieza, aparato o sustancia que se coloca en el cuerpo para mejorar alguna de sus funciones, o con fines estéticos.

Desde la perspectiva del diseño reparamos en esta definición en las palabras aparato, cuerpo, función y estético. Para ello se entregan parámetros de observación desde los elementos cotidianos. Estos conceptos son los que se trabajaron en el Taller Cuerpo. Ahora bien, ¿cómo entendemos esto como Suple? La dimensión de estos conceptos se entendió de cómo se integran entre sí en una estrategia teórico- práctico- visual.

Los alumnos realizaron un dispositivo prótesis con ciertas condiciones en base a un problema concreto: cada grupo debía alcanzar una pelota plástica puesta a 3,2 metros de altura dispuesta en una base en el muro de la Universidad; otra condición entregada en el encargo, fue que el dispositivo se adosara al cuerpo del estudiante, siendo portátil y efectivo y finalmente que la materialidad debía ser cartón y/o materiales afines. 
Gráfico $\mathrm{n}^{\circ} 3$ : El problema de diseño entregado a los estudiantes y las observaciones de lo cotidiano como prótesis.

¿Cómo entendemos PRÓtesis como SUPLE?

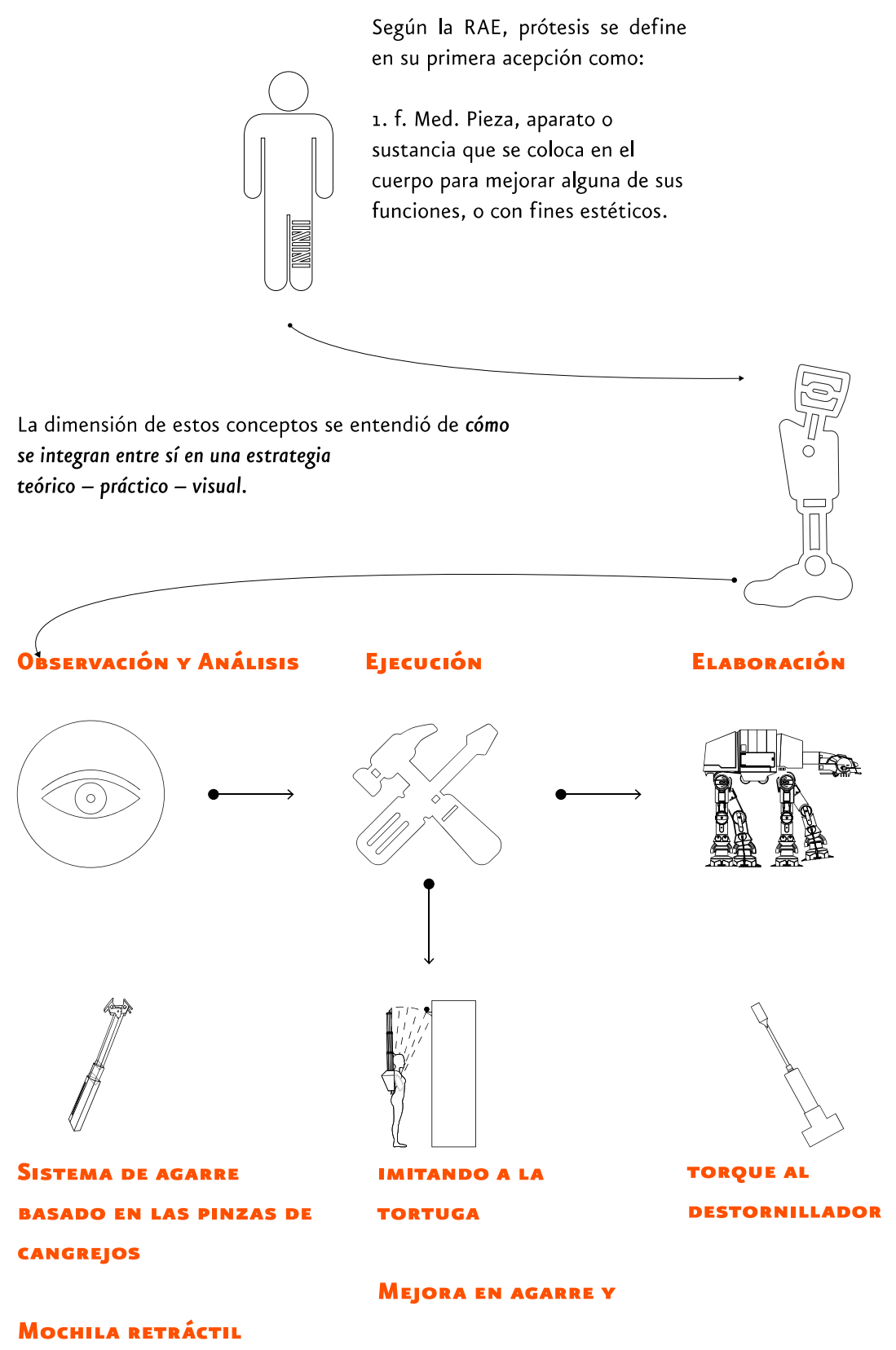

Nota: Infografía de los autores, basados en pictogramas de Noun Proyect (2018). 
Este proceso metodológico de investigación se realizó en base láminas que visualizan el proyecto en etapas y por otra parte en prácticas de pruebas de materiales en terreno, que se fueron modificando e integrando a las propuestas conceptuales presentadas primeramente, generando un discurso equilibrado entre lo conceptual y lo práctico. La experiencia logró el objetivo planteado en la totalidad de los grupos formados y junto a ello una diversidad de soluciones al mismo problema.

Se presenta a continuación, dos ejemplos de los trabajos de los alumnos del Taller Cuerpo de segundo año de la Escuela de Diseño UNIACC.

\section{Proyecto DECÁPODA, corresponde a los estudiantes Leticia Aguilar, Gonzalo} Concha, Israel Díaz, Maite Tobar y Javiera Peralta

La construcción de este insólito dispositivo llevó a los alumnos a estudiar el problema desde las áreas más conceptuales por medio de croquis, para potenciar y fijar la observación, como medio de investigación, mapas de flujos y referentes constructivos hasta las más prácticas, en cuanto a la resistencia de los materiales, sistemas de encajes, de tracción, etc. y cómo este material presentaba diferentes problemas estéticos y formales en su presentación.

\section{Gráfico $\mathrm{n}^{\circ} 4$ Láminas de estudio preliminar y resultado de maqueta final}

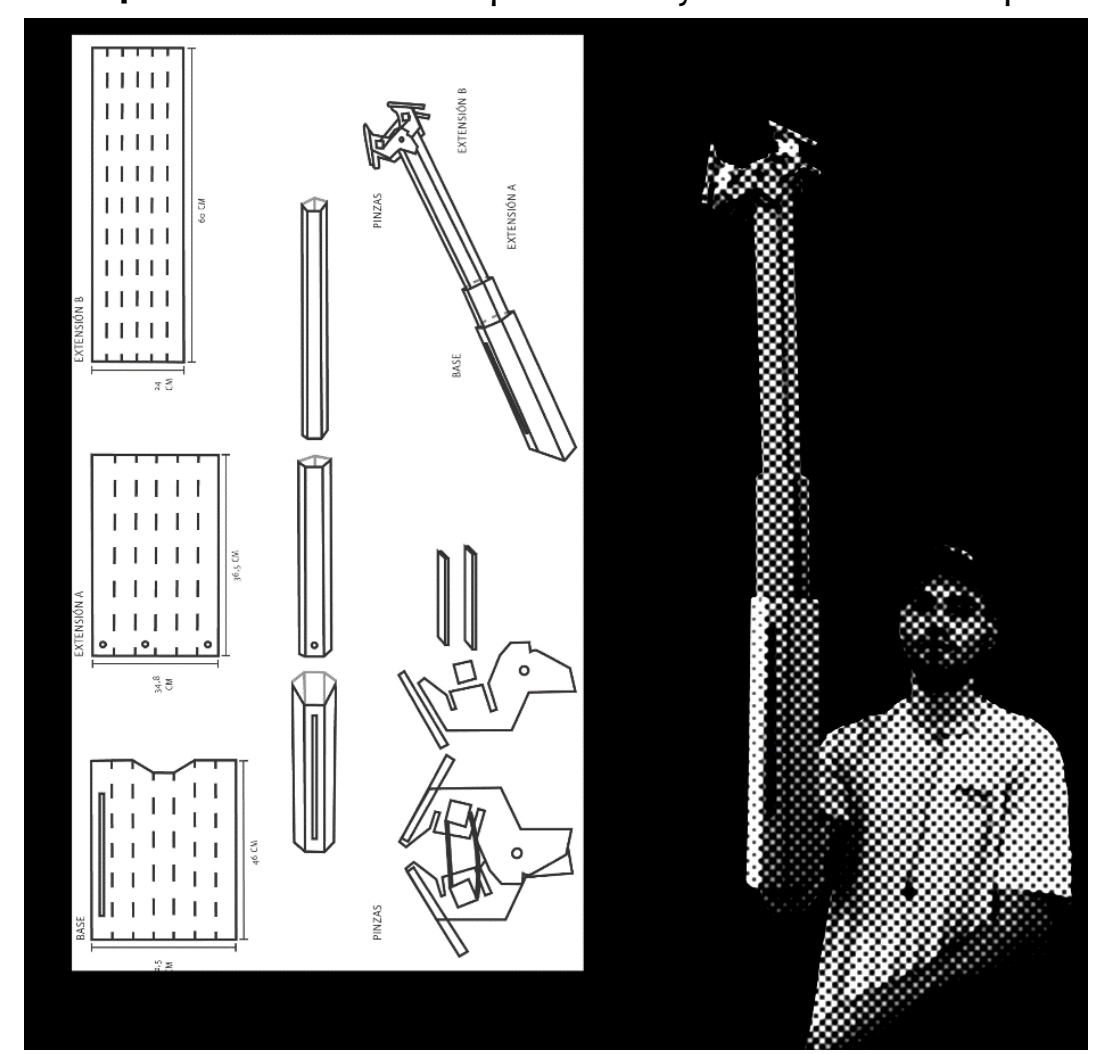

Nota: Dibujos, esquemas, fotografías y maqueta del grupo de estudiantes (2018). 


\section{Proyecto TORTUMOCHI, corresponde a los estudiantes Bárbara Bravo, Cristián} Cares y Eileen Díaz.

Si pensamos en alguna persona que pierde una pierna y debemos realizarle una prótesis, ¿cuál es el objetivo de aquella acción?, ¿una pierna que lo complete o una pierna que lo ayude caminar?, en este caso pensamos que las dos funciones deberían trabajar en armonía y equilibrio. Para ello se trabajó desde la frase con el principio de diseño funcionalista; "La Forma sigue a la Función” y su inversa "La Función Sigue a la Forma".

Gráfico $\mathrm{n}^{\circ} 5$ Láminas de estudio preliminar y resultado de maqueta final

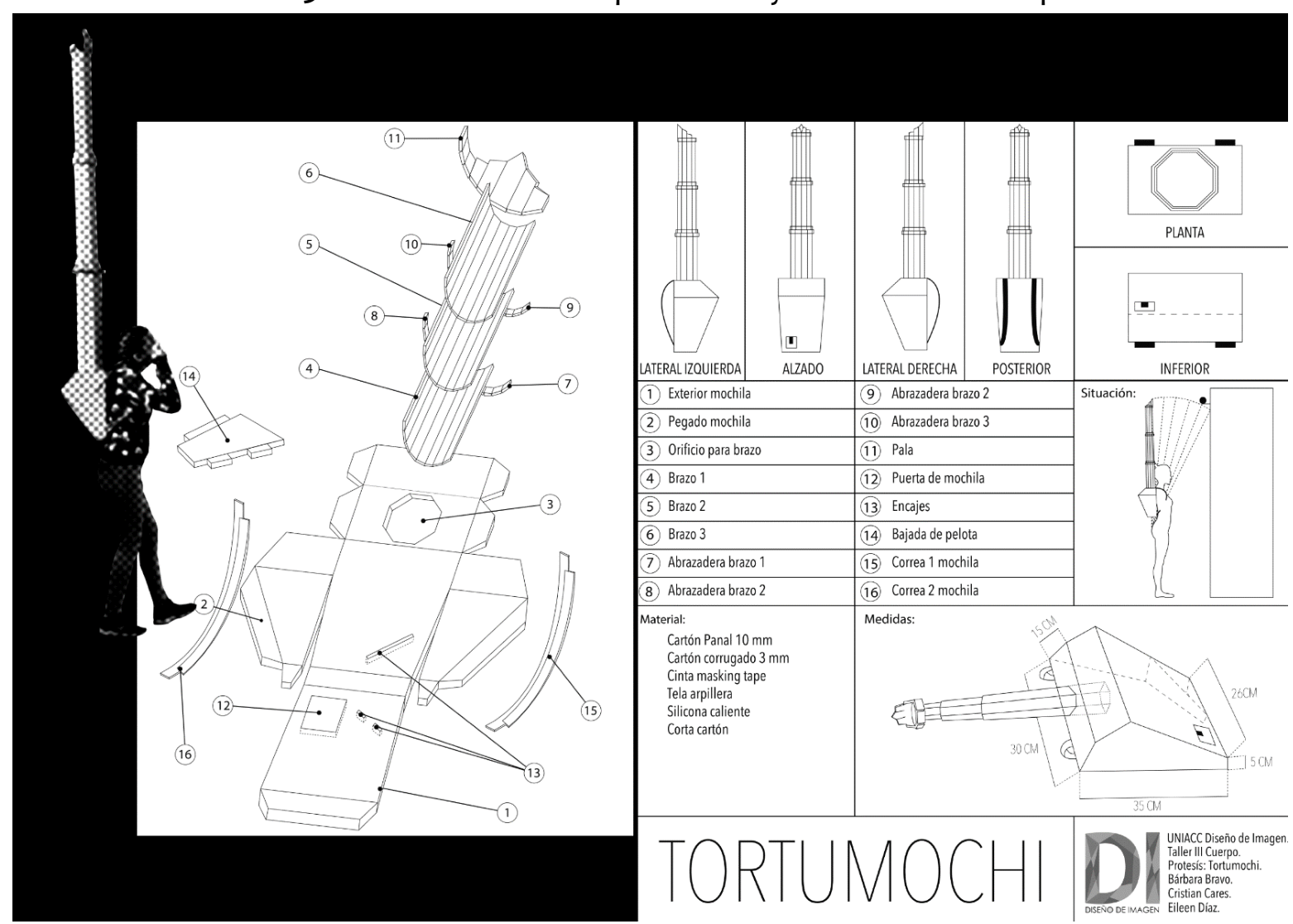

Nota: Dibujos, esquemas, fotografías y maqueta del grupo de estudiantes (2018).

Experiencia de Observación a través de Suple, con profesores de la Escuela de Diseño

Para este número 2 de Suple 2018, la invitación se extiende a 4 docentes de la Escuela de Diseño UNIACC, donde así como en el proyecto entregado a los estudiantes, a estos profesores también se les plantea la problemática, desde la observación como núcleo de la investigación y a sus disciplinas que imparten, con el fin de explorar aquello que en la experiencia de sus oficios se presenten como estos Suples - Prótesis, y cómo estas 
prácticas que se mantiene en la periferia, se convierten en el punto de interés que podrían determinar nuevos métodos, formas, economías y territorios, que la Escuela se ha propuesto investigar dentro del espacio del diseño chileno y sus prácticas contemporáneas.

Los docentes invitados fueron Sandra Hayvel, Dai Liv Fuentes, Patricio González y Lucas Ballocchi

Gráfico n6 Infografía explicativa del territorio del docente y sus disciplinas

\section{relación Suple / Prótesis \\ EN EL TERRITORIO dOMÉstico desde CADA UNA de SUS \\ Disciplinas.}

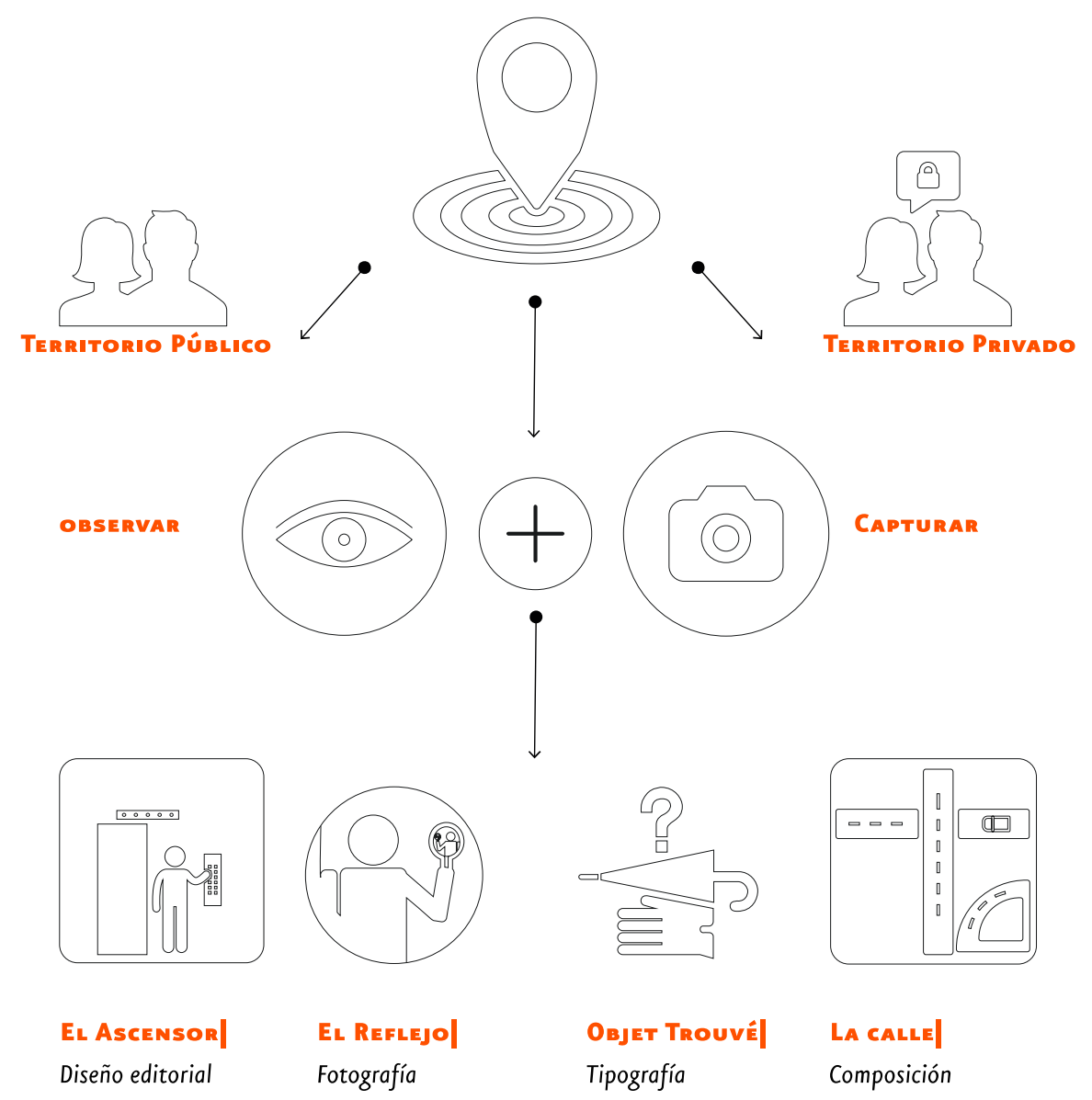

Nota:Infografía de los autores, basados en pictogramas de Noun Proyect (2018). 


\section{Discusión}

Finalizada esta segunda experiencia, en torno a la hipótesis SUPLE para mejorar las prácticas educativas del Diseño, a través de la aplicación de estructuras conceptuales y sistematizadas de observación, se obtienen una serie de respuestas y nuevas interrogantes, esta vez los estudiantes que participaron ya habían trabajado con las fichas de observación Suple, desde sus propios hogares, cuando cursaban primer año, lo que les permitió en esta segunda etapa, comprender más rápidamente la importancia de la observación, lograr una propuesta más creativa y dar cuenta de una solución más rápida a un problema de Diseño más complejo.

La experiencia de este año, permitió obtener resultados de aprendizajes desde las áreas más conceptuales, hasta las más prácticas, por medio del croquis, como método de observación y con el desarrollo de láminas explicativas, como medio de investigación, que contenían mapas de flujos y referentes constructivos con un alto índice de aplicación real.

Por otra parte, al invitar a participar a profesores de esta experiencia, se obtiene una nueva mirada, más profesional, de la observación de lo cotidiano, desde sus propias áreas de trabajo, que sin lugar a dudas, permite concluir que esta fórmula de observación sobre la vida cotidiana es también aplicable a diferentes áreas, no sólo de la educación sino también del diseño de cualquier solución en general. Las creativas y positivas respuestas de las que da cuenta este libro son el testimonio del valor que un sistema metodológico le puede agregar a muchas disciplinas y demuestran, que puede ser usado por diferentes profesionales para el desarrollo de proyectos que mejoren nuestro entorno y la vida social que compartimos.

Fuente: http://www.uniacc.cl/la-universidad/ (2018)

\section{Referencias}

Álvarez, C. Á. (2012). ¿ Qué Sabemos de la Relación entre la Teoría y la Práctica en la Educación?. Revista iberoamericana de educación, 60(2), 12-12.

Arzola, S. (1992). Autoestima y enseñanza media: transferencia pedagógica y calidad de aprendizaje. (Informe No. 1920750) Santiago: FONDECYT.

Arzola, S. (1989). Desarrollo de la autoestima en jóvenes y profesores de enseñanza media. (Informe No. 1890638) Santiago: FONDECYT. 
Arzola, S. (2007). Políticas y Estrategias de Mejoramiento de la Calidad de la Educación. Pensamiento Educativo, 13-29.

Barr, R. \& Tag, J. (1999). De la enseñanza al aprendizaje. Un nuevo paradigma para la educación de pregrado. Universidad de Guadalajara.

Bertillon, A., \& Müller, G. (1889). Instructions for taking descriptions for the identification of criminals and others by the means of anthropometric indications.

Calles, F. (2008). Notas Incómodas sobre la enseñanza del diseño. Foro Alfa. Recuperado el 3 de noviembre de 2006 de http//:foroalfa.com

Chaves, N. (2005). Dos distorsiones en la enseñanza del diseño gráfico. Foro Alfa. Recuperado el 7 de abril de 2007 de http//:foroalfa.com

Freinet, C., \& y Terán, F. J. M. (1985). Freinet: una pedagogía de sentido común. SEP Cultura, Dirección General de Publicaciones.

Freinet, C., \& Rodríguez, H. B. (1996). La escuela moderna francesa; Una pedagogía moderna de sentido común; Las invariantes pedagógicas. Morata.

Gonczi, A., \& Athanasou, J. (1996). Instrumentación de la educación basada en competencias. Perspectivas de la teoría y la práctica en Australia. Competencia laboral y educación basada en normas de competencia, 265-288.

Hagger, P., \& Becket, D. (2000). Bases filosóficas del concepto integrado de competencias. Arguelles. México.

Malpica, M. (1996). El punto de vista pedagógico. Argüelles, A., op. cit, 123-140.

Pibernat, O. (2008). La utilidad en el diseño. Ciclo de exposiciones "Valores del diseño", ddi-cba, Sociedad estatal para el desarrollo del diseño y la innovación, Círculo de Bellas Artes.

Postic, M., \& De Ketele, J. M. (1992). Observar las situaciones educativas (Vol. 61). Narcea Ediciones.

Sánchez, M. (2016). La conceptualización del diseño. Actas de diseño, (20), 237-240. https://thenounproject.com/ 
Alfredo Antonio Da Venezia Vásquez
Licenciado en Bellas Artes, Universidad ARCIS, Productor general del Colectivo
Multidisciplinario de producción cultural NEKOE, Profesor Jornada titular en los
Talleres de Comunicación Visual, de la Escuela Diseño de Imagen, Universidad Uniacc.
Profesor del Taller de los Sentidos, Escuela de Arquitectura Universidad de las
Américas, Viña del Mar. Productor y Coordinador Galería Switch. Escuela de
Arquitectura Universidad de las Américas, Viña del Mar.
Sandra Moreno Oyarzún
Diseñadora Gráfica, mención comunicación, IPS (UTEM). Magister en Gestión
educacional. UDLA. Practitioner en PNL, Programación Neurolingüística. Diseñadora
y Directora de Arte, en las principales revistas del país. Desde 1986 docente Escuelas
de Diseño, en asignaturas de Taller, Tipografía, Color y composición. Directora de
Carrera de Diseño UNIACC. 